\title{
Approximate triangle amplitude for three-body charge exchange processes
}

\author{
E. O. Alt,${ }^{1}$ A. S. Kadyrov, ${ }^{2}$ and A. M. Mukhamedzhanov ${ }^{3}$ \\ ${ }^{1}$ Institut für Physik, Universität Mainz, D-55099 Mainz, Germany \\ ${ }^{2}$ Institute for Nuclear Physics, Ulugbek, Tashkent 702132, Uzbekistan \\ ${ }^{3}$ Cyclotron Institute, Texas A\&M University, College Station, Texas 77843
}

(Received 27 October 1995)

\begin{abstract}
The single-rescattering contribution to the amplitude pertaining to three-body charge exchange reactions (triangle amplitude) contains the off-shell Coulomb $T$-matrix $T^{C}$ describing the intermediate-state Coulomb scattering of charged subsystems. For ease of computation, the latter is usually replaced by the potential $V^{C}$ which, however, is unsatisfactory in many cases. An alternative approximation, obtained by "renormalizing" the "triangle" contribution with $V^{C}$ instead of $T^{C}$ by a simple analytic expression, is shown to yield results in excellent agreement with the numerically calculated exact amplitude, for atomic elastic exchange reactions, over a wide range of (medium to high) projectile energies and scattering angles (including the forward direction).
\end{abstract}

PACS number(s): 34.80.Bm, 34.90.+q, 25.55.Kr, 24.10.-i

In the multiple-scattering formulation of the theory of exchange processes in three-charged-particle systems, the reaction amplitude is given as the sum of the one-particle exchange plus the first- and the higher-order rescattering contributions. At sufficiently high energies one expects that the first two terms of this multiple-scattering series, the socalled "pole" and the triangle amplitude, should provide an adequate description of "diffractive" cross sections which fall off very quickly as one goes away from the extreme forward direction, while the pole amplitude alone is known to be insufficient for achieving a reasonable description of the experimental data, e.g., for electron transfer in electronhydrogen scattering even in the $100-\mathrm{keV}$ range.

The triangle or single-rescattering amplitude contains the off-shell Coulomb $T$ matrix describing intermediate-state Coulomb scattering of the projectile with each of the charged target particles. Though explicitly known, the complicated singularity structure of the latter makes the calculation of such expressions a rather difficult task even today. Hence, in numerical work $T^{C}$ is usually replaced by its Born approximation $V^{C}$, which drastically reduces the required analytical and numerical effort. The approximate exchange amplitude obtained in this way will be called the Coulomb-Born approximation.

Despite its practical importance, we are aware of only two early numerical investigations $[1,2]$ of the exact triangle exchange amplitude. In [2] it was calculated for various electron-transfer processes and compared with the CoulombBorn approximation. The conclusion was that for none of the (limited number of) reactions investigated, the latter could be considered acceptable. Hence, it is evident that an approximation which is much more accurate but not much more expensive to calculate than the Coulomb-Born approximation, would be very useful for practical applications to atomic charge-exchange reactions. We propose here an approximate amplitude which satisfies these requirements. It resulted from an analysis of the analytic properties of the exact triangle amplitude.

Denote the masses and charges of the three particles by $m_{\nu}$ and $e_{\nu}, \nu=1,2,3$. We consider the exchange process $\alpha+(\beta \gamma)_{m} \rightarrow \beta+(\gamma \alpha)_{n}$ : particle $\alpha$, having a center-of-mass (c.m.) momentum $\mathbf{q}_{\alpha}$, impinges on the bound state of particles $\beta$ and $\gamma$ characterized by quantum numbers $m$; in the final state particles $\gamma$ and $\alpha$ are bound in a state with quantum numbers $n$, and particle $\beta$, with a c. m. momentum $\mathbf{q}_{\beta}^{\prime}$, is free. The initial bound-state wave function belonging to the binding energy $\hat{E}_{\alpha m}$ is denoted by $\left|\psi_{\alpha m}\right\rangle$, and analogously for the outgoing bound state. Presently we confine ourselves to the in praxi most important case that the projectile mass $m_{\alpha}$ and the mass of the target particle $\beta$, which the projectile is scattered off, are equal, i.e., $m_{\beta}=m_{\alpha}$.

The triangle contribution to the exchange scattering amplitude is given by $(\gamma \neq \alpha \neq \beta \neq \gamma)$

$$
\mathscr{L}_{\beta n, \alpha m}^{T^{C}}\left(\mathbf{q}_{\beta}^{\prime}, \mathbf{q}_{\alpha}\right)=\left\langle\mathbf{q}_{\beta}^{\prime}\left|\left\langle\psi_{\beta n}\left|T_{\gamma}^{C}(E+i 0)\right| \psi_{\alpha m}\right\rangle\right| \mathbf{q}_{\alpha}\right\rangle
$$

Here, $T_{\gamma}^{C}$ is the Coulomb $T$ operator for the interacting pair $(\alpha \beta)$. On the energy shell the initial- and final-state momenta are related to the energy via

$$
E=\frac{q_{\alpha}^{2}}{2 M_{\alpha}}+\hat{E}_{\alpha m}=\frac{q_{\beta}^{\prime 2}}{2 M_{\beta}}+\hat{E}_{\beta n},
$$

where, e.g., $M_{\alpha}=m_{\alpha}\left(m_{\beta}+m_{\gamma}\right) /\left(m_{\alpha}+m_{\beta}+m_{\gamma}\right)$ is the $\alpha$-channel reduced mass. Similarly, we define the quantity $\mathscr{C}_{\beta n, \alpha m}^{V^{C}}\left(\mathbf{q}_{\beta}^{\prime}, \mathbf{q}_{\alpha}\right)$ which follows from (1) by the replacement $T_{\gamma}^{C} \rightarrow V_{\gamma}^{C}$, and is referred to as the Coulomb-Born approximation of (1). As is well known, for simple bound-state wave functions the latter can even be calculated analytically.

We have investigated [3] the analytic behavior of the exchange amplitude (1) in the $\xi(=\cos \vartheta)$ plane, where $\vartheta=\angle\left(\mathbf{q}_{\beta}^{\prime}, \mathbf{q}_{\alpha}\right)$ is the scattering angle. There we show that the singularity of $\mathscr{C}_{\beta n, \alpha m}^{T^{C}}$ for

$$
\lambda_{\alpha}^{2}\left(\mathbf{q}_{\beta}^{\prime}-\mathbf{q}_{\alpha}\right)^{2}+\left(\kappa_{\beta n}+\kappa_{\alpha m}\right)^{2}=0,
$$

or equivalently at 


$$
\xi=\xi_{(s)}:=\frac{q_{\alpha}^{2}+q_{\beta}^{\prime 2}+\left(\kappa_{\alpha m}+\kappa_{\beta n}\right)^{2} / \lambda_{\alpha}^{2}}{2 q_{\alpha} q_{\beta}^{\prime}}>1,
$$

is the one closest to the physical forward-scattering region. Here, $\quad \kappa_{\alpha m}=\sqrt{2 \mu_{\alpha}\left|\hat{E}_{\alpha m}\right|}, \quad \kappa_{\beta n}=\sqrt{2 \mu_{\beta}\left|\hat{E}_{\beta n}\right|}, \quad$ and $\mu_{\beta}=m_{\alpha} m_{\gamma} /\left(m_{\alpha}+m_{\gamma}\right)$ is the reduced mass of the pair $(\alpha \gamma)$ and analogously for $\mu_{\alpha}\left(=\mu_{\beta}\right.$ in our case $)$, and $\lambda_{\alpha}=\mu_{\alpha} / m_{\alpha}$. Clearly, if its distance to the physical region is small enough, it can dominate completely the near-forward scattering. It is to be noted that $\mathscr{C l}_{\beta n, \alpha m}^{V^{C}}$ has a singularity of similar type at the same position.

We have also derived the corresponding "residues" at this singularity which are, of course, different for the two amplitudes. Let us define the quantities

$$
k_{(s)}^{2}=\lambda_{\alpha}^{2} \frac{\left(q_{\alpha}^{2} \kappa_{\beta n}+q_{\beta}^{\prime 2} \kappa_{\alpha m}\right)}{\left(\kappa_{\beta n}+\kappa_{\alpha m}\right)}+\kappa_{\beta n} \kappa_{\alpha m}
$$

and

$$
p_{(s)}^{2}=\frac{\left(\mathbf{q}_{\beta}^{\prime}+\lambda_{\gamma} \mathbf{q}_{\alpha}\right)^{2} \kappa_{\beta n}+\left(\mathbf{q}_{\alpha}+\lambda_{\gamma} \mathbf{q}_{\beta}^{\prime}\right)^{2} \kappa_{\alpha m}}{\left(\kappa_{\beta n}+\kappa_{\alpha m}\right)}+\kappa_{\beta n} \kappa_{\alpha m},
$$

where $\lambda_{\gamma}=\mu_{\alpha} / m_{\gamma}$ and $\mathbf{q}_{\alpha} \cdot \mathbf{q}_{\beta}^{\prime}$ has still to be substituted by its value $q_{\alpha} q_{\beta}^{\prime} \xi_{(s)}$ at the singularity. Furthermore, we introduce $\xi_{\alpha}^{(s)}=\left[k_{(s)}^{2}+\lambda_{\alpha}^{2} q_{\alpha}^{2}+\kappa_{\alpha m}^{2}\right] / 2 \lambda_{\alpha} k_{(s)} q_{\alpha}$ and $\xi_{\beta}^{(s)}=\left[k_{(s)}^{2}\right.$ $\left.+\lambda_{\alpha}^{2} q_{\beta}^{\prime 2}+\kappa_{\beta n}^{2}\right] / 2 \lambda_{\alpha} k_{(s)} q_{\beta}^{\prime}$. Then we find for the ratio of the exact to the approximate triangle amplitude,

$$
\mathscr{R}_{\beta n, \alpha m}:=\frac{\mathscr{L}_{\beta n, \alpha m}^{T^{C}}\left(\mathbf{q}_{\beta}^{\prime}, \mathbf{q}_{\alpha}\right)}{\mathscr{L}_{\beta n, \alpha m}^{V^{C}}\left(\mathbf{q}_{\beta}^{\prime}, \mathbf{q}_{\alpha}\right)}
$$

in the vicinity of the singularity at (3) or (4)

$$
\mathscr{B}_{\beta n, \alpha m} \stackrel{\xi \rightarrow \xi_{(s)}}{\approx} \mathscr{R}_{\beta n, \alpha m}^{\text {sing }}
$$

$$
:=\frac{A^{i \eta_{\gamma}^{(s)} \tilde{\mathscr{C}}_{\beta n, \alpha m}^{\operatorname{sing}}}}{\left[\lambda_{\alpha}^{2}\left(\mathbf{q}_{\beta}^{\prime}-\mathbf{q}_{\alpha}\right)^{2}+\left(\kappa_{\beta n}+\kappa_{\alpha m}\right)^{2}\right]^{-2 i \eta_{\gamma}^{(s)}}}
$$

with

$$
\tilde{\mathscr{B}}_{\beta n, \alpha m}^{\text {sing }}=C_{0}^{2} \frac{\Gamma\left(1-\eta_{\alpha m}\right)}{\Gamma\left(1-\eta_{\alpha m}-i \eta_{\gamma}^{(s)}\right)} \frac{\Gamma\left(1-\eta_{\beta n}\right)}{\Gamma\left(1-\eta_{\beta n}-i \eta_{\gamma}^{(s)}\right)} \frac{\Gamma\left(-\eta_{\alpha m}-\eta_{\beta n}-2 i \eta_{\gamma}^{(s)}\right)}{\Gamma\left(-\eta_{\alpha m}-\eta_{\beta n}\right)}
$$

and

$$
A=\left(\frac{\eta_{\gamma}^{(s)} k_{(s)}}{2 m_{\alpha} \lambda_{\alpha}^{2} e_{\alpha} e_{\beta} p_{(s)}}\right)^{2} \frac{\sqrt{\left(\xi_{\alpha}^{(s) 2}-1\right)\left(\xi_{\beta}^{(s) 2}-1\right)}}{q_{\alpha} q_{\beta}^{\prime}\left(\xi_{(s)}^{2}-1\right)} .
$$

Here, $\eta_{\alpha m}=e_{\beta} e_{\gamma} \mu_{\alpha} / \kappa_{\alpha m}$ is the Coulomb parameter for the incoming bound-state $(\beta \gamma)_{m}$, with $\eta_{\beta n}$ being defined analogously. For atomic processes involving hydrogenic bound state wave functions, we have $\eta_{\alpha m}=-n_{\alpha m}$ and $\eta_{\beta n}=-n_{\beta n}$ with $n_{\alpha m}\left(n_{\beta n}\right)$ being the corresponding principal quantum number. Furthermore, $\eta_{\gamma}^{(s)}=e_{\alpha} e_{\beta} \sqrt{m_{\alpha} / 4\left(E+i 0-k_{(s)}^{2} / 2 M_{\gamma}\right)}$ is the Coulomb parameter appropriate for the intermediate-state scattering, and $C_{0}^{2}=2 \pi \eta_{\gamma}^{(s)} /\left(\exp \left\{2 \pi \eta_{\gamma}^{(s)}\right\}-1\right)$ the Coulomb penetration factor. $\Gamma(z)$ is the gamma function.

From this we deduce the following results which are valid for attractive and repulsive Coulomb scattering in intermediate state.

(i) For three-body energies, $E>k_{(s)}^{2} / 2 M_{\gamma}$, the magnitude of the ratio of the exact to the approximate triangle amplitude directly at the singular point (4) is given by

$$
\lim _{\xi \rightarrow \xi_{(s)}}\left|\mathscr{B}_{\beta n, \alpha m}\right|=\left|\tilde{\mathscr{B}}_{\beta n, \alpha m}^{\operatorname{sing}}\right| .
$$

That is, in some neighborhood of (4), we have the simple relation

$$
\left|\mathscr{C l}_{\beta n, \alpha m}^{T^{C}}\left(\mathbf{q}_{\beta}^{\prime}, \mathbf{q}_{\alpha}\right)\right| \approx\left|\tilde{\mathscr{R}}_{\beta n, \alpha m}^{\text {sing }}\right|\left|\mathscr{L}_{\beta n, \alpha m}^{V^{C}}\left(\mathbf{q}_{\beta}^{\prime}, \mathbf{q}_{\alpha}\right)\right| .
$$

As follows from (9), only for sufficiently small $\eta_{\gamma}^{(s)}$, i.e., for sufficiently large energies, $\left|\tilde{\mathscr{R}}_{\beta n, \alpha m}^{\text {sing }}\right|$ approaches the value of one, implying that the Coulomb-Born approximation can be considered satisfactory. For $E<k_{(s)}^{2} / 2 M_{\gamma}$, when $\eta_{\gamma}^{(s)}$ is purely imaginary, $\left|\tilde{\mathscr{R}}_{\beta n, \alpha m}^{\text {sing }}\right|$ is a somewhat more complicated function.

By definition, Eq. (12) holds for $\xi \approx \xi_{(s)}>1$. But it is natural to conjecture that its range of validity may extend - as an approximate relation-into the physical region, at least in some neighborhood of the forward direction, provided $\xi_{(s)}$ is not too far off the value of one.

To simplify the discussion, we consider only elastic exchange with particles $\alpha$ and $\beta$ being identical [as in $\left(e, e^{\prime}\right)$ or $\left(p, p^{\prime}\right)$ reactions], i.e., $n=m$ and $\hat{E}_{\beta m}=\hat{E}_{\alpha m}$. Use of the on-shell condition allows us to express Eq. (4) as

$$
\xi_{(s)}=1+2 \frac{\left(1+2 m_{\alpha} / m_{\gamma}\right)\left|\hat{E}_{\alpha m}\right|}{E+\left|\hat{E}_{\alpha m}\right|}
$$

Clearly, $\xi_{(s)} \rightarrow 1$ for $E \rightarrow \infty$, irrespective of masses and of the binding energy; hence relation (12) can be expected to be valid even for physical values of $\xi=\cos \vartheta$ (but our nonrelativistic theory may have ceased to be appropriate then). For intermediate energies, if the projectile mass is much larger than the mass of the spectator $\gamma\left(m_{\alpha} / m_{\gamma} \gg 1\right)$, we have 
TABLE I. Comparison of $\left|\mathscr{R}_{\beta 0, \alpha 0}\right|$ obtained by quadrature as function of the cosine of the scattering angle, with $\left|\mathscr{R}_{\beta 0, \alpha 0}^{\text {sing }}\right|$, for several projectile kinetic energies, for the reaction $e+\mathrm{H}(1 s) \rightarrow e^{\prime}+\mathrm{H}(1 s)$. Also shown is the corresponding value of $\xi_{(s)}$.

\begin{tabular}{ccccc}
\hline \hline & $0.1 \mathrm{keV}$ & $1 \mathrm{keV}$ & $10 \mathrm{keV}$ & $100 \mathrm{keV}$ \\
$\left(\xi_{(s)}\right)$ & $(1.2723)$ & $(1.0272)$ & $(1.0027)$ & $(1.0003)$ \\
$\left|\mathscr{R}_{\beta 0, \alpha 0}^{\text {sing }}\right|$ & 0.1363 & 0.6661 & 0.8876 & 0.9637 \\
$\xi=\cos \vartheta$ & & \multicolumn{2}{c}{$\left|\mathscr{B}_{\beta 0, \alpha 0}\right|$} & \\
\hline 1.0000 & 0.1337 & 0.6633 & 0.8877 & 0.9637 \\
0.9239 & 0.1765 & 0.6567 & 0.8860 & 0.9635 \\
0.7071 & 0.2900 & 0.6634 & 0.8862 & 0.9635 \\
0.3827 & 0.4190 & 0.6920 & 0.8897 & 0.9639 \\
0.0000 & 0.5260 & 0.7259 & 0.8946 & 0.9645 \\
-0.3827 & 0.6024 & 0.7554 & 0.8992 & 0.9650 \\
-0.7071 & 0.6516 & 0.7771 & 0.9031 & 0.9655 \\
-0.9239 & 0.6780 & 0.7898 & 0.9053 & 0.9658 \\
-1.0000 & 0.6873 & 0.7939 & 0.9062 & 0.9659 \\
\hline \hline
\end{tabular}

$\xi_{(s)} \gg 1$ so that the right-hand side (rhs) of (12) will not yield satisfactory results for $\xi \leqslant 1$. A typical example is $\mathrm{H}\left(p, p^{\prime}\right) \mathrm{H}$ for which $m_{\alpha} / m_{\gamma}=m_{p} / m_{e}$. On the other hand, for $m_{\alpha} / m_{\gamma} \ll 1$, as it happens in reactions like $\mathrm{H}\left(e, e^{\prime}\right) \mathrm{H}$ where $m_{\alpha} / m_{\gamma}=m_{e} / m_{p}, \xi_{(s)}$ can be very close to one provided the energy is not small. In such situations relation (12) is expected to represent a reliable approximation in the physical region already for moderately high energies.

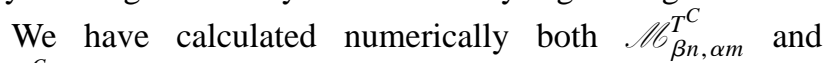
$\mathscr{L}_{\beta n, \alpha m}^{V^{C}}$ for physical values of $\xi$. Thus the absolute value of their ratio $\left|\mathscr{R}_{\beta n, \alpha m}\right|$ can be compared with its value $\left|\tilde{\mathscr{R}}_{\beta n, \alpha m}^{\text {sing }}\right|$ at the singular point $\xi_{(s)}$ in the unphysical region, cf. Eq. (11). This is done in Table I for the elastic exchange reaction $e+\mathrm{H}(1 s) \rightarrow e^{\prime}+\mathrm{H}(1 s)$, i.e., $m=n=0$, with the index zero characterizing hydrogen atoms in the ground state. Inspection reveals that for electron energies even as low as $1 \mathrm{keV}$ the numerical values are reproduced to an excellent accuracy for angles up to, say, $60^{\circ}$, while from 10 $\mathrm{keV}$ on the validity of relation (12) practically extends over the whole angle regime. The reason for this success is twofold. First, in the range of energies and scattering angles considered the absolute value of $\mathscr{C l}_{\beta n, \alpha m}^{T^{C}}$ differs very little from that of $\mathscr{C l}_{\beta n, \alpha m}^{V^{C}}$ with respect to their angular dependence. Second, these two quantities differ noticeably with respect to their magnitude. But this defect is cured by the (angle-independent) "renormalization" factor $\left|\tilde{\mathscr{R}}_{\beta n, \alpha m}^{\text {sing }}\right|$ which, as indicated above, is not so surprising in view of the closeness of the position $\xi_{(s)}$ of the singular point to the physical region. For $p+\mathrm{H}(1 s) \rightarrow p^{\prime}+\mathrm{H}(1 s)$, for which the analogous results are shown in Table II, the rhs of (12) yields similarly accurate results but only beginning at higher energies (this latter fact is as expected since here $\xi_{(s)}$ is much larger than its value for the electron reaction at the same energy). Consequently, whenever the triangle amplitude alone suffices to describe exchange cross sections (and the energy is larger than, say, $1 \mathrm{keV}$ for electron-induced and 50 $\mathrm{keV}$ for proton-induced reactions), the easy-to-calculate approximate formula (12) can be utilized which will greatly simplify calculations.

(ii) Encouraged by these results we suggest the relation

$$
\mathscr{C}_{\beta n, \alpha m}^{T^{C}}\left(\mathbf{q}_{\beta}^{\prime}, \mathbf{q}_{\alpha}\right) \approx \mathscr{R}_{\beta n, \alpha m}^{\text {sing }} \mathscr{C l}_{\beta n, \alpha m}^{V^{C}}\left(\mathbf{q}_{\beta}^{\prime}, \mathbf{q}_{\alpha}\right)
$$

expressing the exact rescattering amplitude itself by the ap-

TABLE II. Same as in Table I, but for the reaction $p+\mathrm{H}(1 s) \rightarrow p^{\prime}+\mathrm{H}(1 s)$.

\begin{tabular}{ccccc}
\hline \hline & $0.01 \mathrm{MeV}$ & $0.1 \mathrm{MeV}$ & $1 \mathrm{MeV}$ & $10 \mathrm{MeV}$ \\
$\left(\xi_{(s)}\right)$ & $(10.9902)$ & $(1.9990)$ & $(1.0999)$ & $(1.0010)$ \\
$\left|\mathscr{R}_{\beta 0, \alpha 0}^{\text {sing }}\right|$ & $0.3602(-2)$ & 0.2504 & 0.6841 & 0.8923 \\
$\xi=\cos \vartheta$ & & $\left|\mathscr{\beta}_{\beta 0, \alpha 0}\right|$ & & \\
\hline 1.0000 & $0.4235(-2)$ & 0.2572 & 0.6861 & 0.8926 \\
0.9239 & $0.4238(-2)$ & 0.2574 & 0.6864 & 0.8926 \\
0.7071 & $0.4246(-2)$ & 0.2578 & 0.6866 & 0.8924 \\
0.3827 & $0.4257(-2)$ & 0.2583 & 0.6864 & 0.8922 \\
0.0000 & $0.4269(-2)$ & 0.2587 & 0.6861 & 0.8921 \\
-0.3827 & $0.4280(-2)$ & 0.2589 & 0.6857 & 0.8920 \\
-0.7071 & $0.4290(-2)$ & 0.2590 & 0.6855 & 0.8919 \\
-0.9239 & $0.4322(-2)$ & 0.2591 & 0.6853 & 0.8919 \\
-1.0000 & 0.6063 & 0.6382 & 0.7891 & 0.9099 \\
\hline \hline
\end{tabular}


TABLE III. The lhs of relation (15) calculated as function of the cosine of the scattering angle, in comparison with the rhs, for two projectile kinetic energies, for the reaction $e+\mathrm{H}(1 s) \rightarrow e^{\prime}+\mathrm{H}(1 s)$. Values of $\xi_{(s)}$ as in Table I.

\begin{tabular}{ccc}
\hline \hline & $10 \mathrm{keV}$ & $100 \mathrm{keV}$ \\
$\left(\xi_{(s)}\right)$ & $(1.0027)$ & $(1.0003)$ \\
$\operatorname{rhs}$ & $0.7300-i 0.5049$ & $0.9350-i 0.2335$ \\
$\xi=\cos \vartheta$ & $\mathrm{lhs}$ & \\
\hline 1.0000 & $0.7323-i 0.5018$ & $0.9354-i 0.2322$ \\
0.9239 & $0.7032-i 0.5389$ & $0.9257-i 0.2671$ \\
0.7071 & $0.6870-i 0.5597$ & $0.9221-i 0.2794$ \\
0.3827 & $0.6811-i 0.5725$ & $0.9205-i 0.2861$ \\
0.0000 & $0.6745-i 0.5819$ & $0.9198-i 0.2903$ \\
-0.3827 & $0.6797-i 0.5887$ & $0.9195-i 0.2930$ \\
-0.7071 & $0.6804-i 0.5936$ & $0.9194-i 0.2947$ \\
-0.9239 & $0.6813-i 0.5963$ & $0.9194-i 0.2956$ \\
-1.0000 & $0.6815-i 0.5973$ & $0.9195-i 0.2958$ \\
\hline \hline
\end{tabular}

proximate one. Note that $\mathscr{C l}_{\beta n, \alpha m}^{V^{C}}$ is purely real. For a detailed test of relation (14) it is preferable to divide both sides by $\mathscr{C l}_{\beta n, \alpha m}^{V^{C}}$ and by the oszillating factor $\left[\lambda_{\alpha}^{2}\left(\mathbf{q}_{\beta}^{\prime}-\mathbf{q}_{\alpha}\right)^{2}\right.$

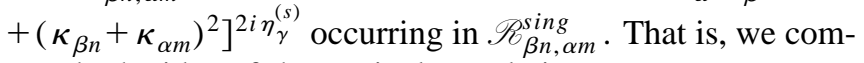
pare both sides of the equivalent relation

$$
\begin{gathered}
\mathscr{R}_{\beta n, \alpha m}\left[\lambda_{\alpha}^{2}\left(\mathbf{q}_{\beta}^{\prime}-\mathbf{q}_{\alpha}\right)^{2}+\left(\kappa_{\beta n}+\kappa_{\alpha m}\right)^{2}\right]^{-2 i \eta_{\gamma}^{(s)}} \\
\approx A^{i \eta_{\gamma}^{(s)} \tilde{\mathscr{R}}_{\beta n, \alpha m}^{\operatorname{sing}} .}
\end{gathered}
$$

Recall that the lhs which combines all angle-dependent factors is obtained by quadrature without any approximation. The results are shown in Table III for electron, and in Table IV for proton exchange scattering off hydrogen atoms in their ground state, at two values of the projectile energy. Inspection reveals that both sides agree with each other to within a few percent with respect to their real, and to a somewhat lesser accuracy also with respect to their imaginary parts, over a wide regime of energies and scattering angles. This latter fact is understandable: the imaginary part of the full triangle amplitude, $\mathscr{C l}_{\beta n, \alpha m}^{T^{C}}$, is caused by the unitarity cut, due to all the terms in the expansion of $T_{\gamma}^{C}$ of order higher than the Born approximation $V_{\gamma}^{C}$ which makes up the real amplitude $\mathscr{C l}_{\beta n, \alpha m}^{V^{C}}$. Therefore, their contribution to $\operatorname{Im} \mathscr{C l}_{\beta n, \alpha m}^{T^{C}}$ cannot be expected to be so reliably represented by the imaginary part of $\mathscr{B}_{\beta n, \alpha m}^{s i n g}$. Nevertheless, if the accuracy of the approximation (14) is sufficient the rhs of (14) can be used for the exact exchange amplitude which will lead to considerable simplifications of calculations. Clearly, the higher the energy is the more reliable this approximation will be.

Two final comments are appropriate. (i) For a given energy, the smaller the binding energy $\left|\hat{E}_{\alpha m}\right|$ is, the closer to the physical region lies $\xi_{(s)}$ and, thus, the larger is the range of parameters for which (12) and (14) represent excellent approximations. This has been verified at the example of the reaction $e+\mathrm{H}(2 s) \rightarrow e^{\prime}+\mathrm{H}(2 s)$. (ii) The quality of the approximation formulas (12) and (14) for bound-state excitation $(n>m)$ cannot be expected to be as generally good as for elastic exchange $(n=m)$. Two opposing tendencies come into play: as before, $\xi_{(s)}$ is located the closer to one the larger $n$ is; on the other hand, for excitation the Coulomb-Born approximation becomes at intermediate energies very much

TABLE IV. Same as in Table III, but for the reaction $p+\mathrm{H}(1 s) \rightarrow p^{\prime}+\mathrm{H}(1 s)$. Values of $\xi_{(s)}$ as in Table II.

\begin{tabular}{ccc}
\hline \hline & $1 \mathrm{MeV}$ & $10 \mathrm{MeV}$ \\
$\left(\xi_{(s)}\right)$ & $(1.0999)$ & $(1.0010)$ \\
$\mathrm{rhs}$ & $-0.6791-i 0.0823$ & $0.3917-i 0.8018$ \\
$\xi=\cos \vartheta$ & $\mathrm{lhs}$ & \\
\hline 1.0000 & $-0.6793-i 0.0959$ & $0.3955-i 0.8002$ \\
0.9239 & $-0.6806-i 0.0888$ & $0.3754-i 0.8098$ \\
0.7071 & $-0.6822-i 0.0780$ & $0.3573-i 0.8177$ \\
0.3827 & $-0.6824-i 0.0743$ & $0.3482-i 0.8215$ \\
0.0000 & $-0.6811-i 0.0824$ & $0.3468-i 0.8219$ \\
-0.3827 & $-0.6775-i 0.1063$ & $0.3532-i 0.8191$ \\
-0.7071 & $-0.6681-i 0.1534$ & $0.3696-i 0.8117$ \\
-0.9239 & $-0.6393-i 0.2469$ & $0.4050-i 0.7946$ \\
-1.0000 & $-0.3316-i 0.7160$ & $0.7556-i 0.5069$ \\
\hline
\end{tabular}


smaller in forward direction than the exact triangle amplitude, due to the near orthogonality of the wave functions for the incoming and the outgoing bound states. However, calculations show that for scattering angles larger than $10^{\circ}-$ $20^{\circ}$ the nonorthogonality effects become negligible and thus our approximation works well also for excitation. This problem is discussed in Ref. [3].

This work was supported in part by the Deutsche Forschungsgemeinschaft, Project No. 436 USB-113-1-0 and by the DOE under Grant No. DE-FG03-93ER40773.
[1] C. S. Shastry, L. Kumar, and J. Callaway, Phys. Rev. A 1, 1137 (1970).

[2] J. Y. C. Chen and P. J. Kramer, Phys. Rev. A 5, 1207
(1972).

[3] E. O. Alt, A. S. Kadyrov, and A. M. Mukhamedzhanov (unpublished). 